\section{Should Azithromycin No Longer Be Considered a Drug of Choice for Community- Acquired Pneumonia Because of Its Potential to Cause Cardiovascular Death?}

\section{THE "PRO" SIDE}

The current Canadian and US guidelines for communityacquired pneumonia (CAP) recommend azithromycin alone in mild cases and azithromycin in combination with a ß-lactam for patients admitted to hospital with moderate to severe CAP. ${ }^{1,2}$ To demonstrate that azithromycin should not be considered an alternative for the treatment of CAP, a medication assessmentbased on indication, effectiveness, and safety-will be completed.

\section{Is Azithromycin Indicated for the Treatment of CAP?}

The above-mentioned guidelines recommend that azithromycin be used alone or together with a ß-lactam to ensure coverage for "atypical" organisms, that is, Mycoplasma pneumoniae, Chlamydophila pneumoniae, and Legionella spp. The guidelines themselves acknowledge that the evidence for empiric coverage is rather poor, and the European guidelines do not recommend empiric coverage for atypical organisms. ${ }^{3}$ Although a recent analysis of the British Thoracic Society national audits demonstrated reduced mortality (odds ratio [OR] 0.72, 95\% confidence interval [CI] 0.60-0.85) among patients treated with dual therapy relative to those treated with B-lactam alone, that analysis was retrospective. ${ }^{4}$ The retrospective design has many limitations, the most important among them being selection bias. In a recent meta-analysis, the Cochrane Collaboration analyzed randomized controlled trials involving adult patients admitted to hospital because of CAP, comparing antibiotic regimens with coverage of atypical organisms (e.g., macrolides, fluoroquinolones, tetracyclines) with regimens without atypical coverage (e.g., amoxicillin, penicillin, ceftriaxone). ${ }^{5}$ A total of 28 trials involving 5939 patients were analyzed. Although there was an advantage in bacteriologic eradication in the arm with coverage of atypical organisms, this advantage disappeared when only high-quality studies were included in the analysis. Overall, the authors concluded that there was no benefit in terms of survival or clinical efficacy (defined as rate of clinical failure; e.g., lack of clinical improvement, deterioration, relapse, and/or modifica- tion of the antibiotic treatment). From this evidence, it can be concluded that azithromycin providing empiric coverage of atypical organisms is not indicated.

\section{Is Azithromycin Effective in the Treatment of CAP?}

Azithromycin may be effective in eradicating atypical organisms, but such bacteria are the causative organism in less than $20 \%$ of cases of pneumonia. The most common organism associated with CAP is Streptococcus pneumoniae. Since the 1990s, resistance to macrolides has been reported in $S$. pneumoniae isolates, and rates of resistance have increased since then: from $3.7 \%$ in 1995 to $19 \%$ in 2005.6 Currently, local resistance rates of up to $25 \%$ have been reported (antimicrobial susceptibility report for 2011, St Michael's Hospital, Toronto, Ontario) With this level of resistance, there are concerns about the utility of azithromycin in the treatment of CAP. From this evidence, it can be concluded that there is a risk of potential treatment failure through lack of efficacy.

\section{Is Azithromycin Safe to Use in the Treatment of CAP?}

For many practitioners, azithromycin has been the "go to" macrolide, as it was considered "safe". It had not been associated with certain cardiovascular toxicities, in particular torsades de pointes, and potential drug interactions, as have been described for clarithromycin and erythromycin. However, recent literature has raised questions about the safety of azithromycin. Case reports of torsades de pointes associated with azithromycin have been published, and AZCERT (an independent nonprofit organization with a mandate to provide credible information on drugs that prolong the QT interval) now lists it as a medication with substantial evidence that it causes this problem. ${ }^{7-9}$

A recent retrospective cohort study using the Tennessee Medicaid database reported an increase in cardiovascular mortality associated with azithromycin use. ${ }^{10}$ In that study, a cohort of patients who took azithromycin was compared with a propensity score-matched cohort of patients who took no antibiotics. To control for confounding by indication, amoxicillin was used as a control group (not propensity score-matched). The matched cohorts were similar in terms of age (48 years), sex ratio ( $77 \%$ female), concomitant use of cardiovascular medications (angiotensin-converting enzyme [ACE] inhibitors 28\%, B-blockers 21\%, loop diuretics 17\%), 
and risk for cardiovascular disease (mean summary score for risk of cardiovascular disease 9.2). Compared with no antibiotic treatment, during the 5-day period of azithromycin use there was an increased risk of cardiovascular death (hazard ratio 2.88, 95\% CI 1.79-4.63) and death from any cause (hazard ratio 1.85 , 95\% CI 1.25-2.75). Use of amoxicillin was not associated with an increased risk. The risk of cardiovascular death was substantially increased among those with the greatest decile of cardiovascular risk score. In that group there was one excess cardiovascular death for every 4100 azithromycin prescriptions.

In response to this trial, a cohort study conducted in Denmark compared propensity score-matched cohorts of azithromycin recipients and patients who had not received antibiotics; an unmatched cohort of patients who received penicillin V was used as a control group. ${ }^{11}$ Compared with the earlier study, these cohorts were much younger (39 years), and fewer of the patients used ACE inhibitors (6\%), ß-blockers $(5 \%)$, and loop diuretics (2\%). Compared with no antibiotic use, there was an increased risk of death from cardiovascular causes (rate ratio $2.85,90 \%$ CI 1.13-7.24) within the cohort that used azithromycin. In the comparison of azithromycin and penicillin $\mathrm{V}$ cohorts, the propensity score-adjusted rates were no different (rate ratio $0.93,95 \%$ CI $0.56-1.55$ ).

Given the observational nature of both studies, there are significant limitations. The contrast in results might be explained by differences in the populations studied. The Danish trial enrolled a younger cohort with lower cardiovascular risk. This difference in cardiovascular risk is the most important potential limitation, considering that the probable cause of increased cardiovascular death was proarrhythmia (torsades de pointes). Risk factors for torsades de pointes include heart disease (heart failure and acute myocardial infarction), advanced age, female sex, treatment with diuretics, and bradycardia. These factors were more likely to be present in the older Tennessee cohort, which had greater use of medications, including ß-blockers and loop diuretics. Nonetheless, despite the limitations of the observational studies, it can be concluded that azithromycin is not safe.

\section{Conclusion}

It may be some time before the major societies update their guidelines, but individual institutions and health regions should re-evaluate their CAP guidelines with the goal of removing azithromycin from treatment algorithms, particularly for inpatient use. Hospital inpatients are typically older individuals, often with pre-existing cardiac disease, who are taking multiple medications and are at particular risk for the proarrhythmic effects of azithromycin. If azithromycin is to be used in selected patients, a protocol should be in place to identify how those individuals should be monitored and what degree of QTc prolongation would be tolerated before the drug is discontinued.
However, the best approach would be to not use a drug that is not indicated, not effective, and not safe.

\section{References}

1. Mandell LA, Marrie TJ, Grossman RF, Chow AW, Hyland RH; CAP Working Group. Summary of the Canadian guidelines for the initial management of community-acquired pneumonia: an evidence-based update by the Canadian Infectious Disease Society and the Canadian Thoracic Society. Can Respir J. 2000;7(5):371-82.

2. Mandell LA, Wunderink RG, Anzueto A, Bartlett JG, Campbell GD, Dean NC, et al. Infectious Diseases Society of America/American Thoracic Society consensus guidelines on the management of community-acquired pneumonia in adults. Clin Infect Dis. 2007:44 Suppl 2:S27-72.

3. Woodhead M, Blasi F, Ewig S, Garau J, Huchon G, Leven M, et al.; Joint Taskforce of the European Respiratory Society and European Society for Clinical Microbiology and Infectious Diseases. Guidelines for the management of adult lower respiratory tract infections-full version. Clin Microbiol Infect. 2011;17 Suppl 6:E1-59.

4. Rodrigo C, Mckeever TM, Woodhead M, Lim WS; British Thoracic Society. Single versus combination antibiotic therapy in adults hospitalised with community acquired pneumonia. Thorax. 2013;68(5):493-5.

5. Eliakim-Raz N, Robenshtok E, Shefet D, Gafter-Gvili A, Vidal L, Paul M, et al. Empiric antibiotic coverage of atypical pathogens for communityacquired pneumonia in hospitalized adults. Cochrane Database Syst Rev. 2012;9:CD004418.

6. Karlowsky JA, Lagacé-Wiens PR, Low DE, Zhanel GG. Annual macrolide prescription rates and the emergence of macrolide resistance among Streptococcus pneumoniae in Canada from 1995 to 2005. Int J Antimicrob Agents. 2009;34(4):375-9.

7. Huang BH, Wu CH, Hsia CP, Yin Chen C. Azithromycin-induced torsade de pointes. Pacing Clin Electrophysiol. 2007;30(12):1579-82.

8. Kezerashvili A, Khattak H, Barsky A, Nazari R, Fisher JD. Azithromycin as a cause of QT-interval prolongation and torsade de pointes in the absence of other known precipitating factors. I Interv Card Electrophysiol. 2007;18(3):243-6.

9. Drugs that prolong the QT interval and/or induce torsade de pointes. In: CredibleMeds [website]. Oro Valley (AZ): AZCERT Inc; 2013 [cited 2013 Jul 20]. Available from: www.azcert.org/medical-pros/drug-lists/ list-01.cfm?sort=Generic_name

10. Ray WA, Murray KT, Hall K, Arbogast PG, Stein CM. Azithromycin and the risk of cardiovascular death. $N$ Engl J Med. 2012:366(20):1881-90.

11. Svanström H, Pasternak B, Hviid A. Use of azithromycin and death from cardiovascular causes. N Engl J Med. 2013;368(18):1704-12.

Heather Kertland, PharmD, FCSHP

Clinical Pharmacy Specialist/Leader

St Michael's Hospital

Assistant Professor

Leslie Dan Faculty of Pharmacy

University of Toronto

Toronto, Ontario

Competing interests: None declared.

Acknowledgement: I would like to thank Linda Dresser, PharmD, FCSHP, for reviewing the draft of this paper and for her helpful insights on the debate.

\section{THE "CON" SIDE}

Azithromycin has been available for over 20 years, and millions of patients have safely received the drug orally or parenterally for the treatment of respiratory tract infections. However, in recent years, the cardiac safety of azithromycin has 
been questioned, primarily because of increasing concerns regarding the potential for QT interval prolongation, torsades de pointes, and cardiovascular death. Yet despite abundant clinical use, only a small number of cases of QT prolongation, torsades de pointes, and polymorphic ventricular tachycardia associated with azithromycin have been reported. ${ }^{1-6}$ All of these patients had additional, well-known risk factors for QT prolongation and torsades de pointes, including concomitant drugs (amiodarone, methadone), left ventricular dysfunction, hypokalemia, advanced age, and bradycardia. A review of the US Food and Drug Administration Adverse Event Reporting System for 2004 to 2007 identified only 20 cases of torsades de pointes associated with azithromycin, compared with 32 cases associated with ciprofloxacin, a drug considered safe from a cardiac standpoint, and 61 cases associated with levofloxacin.?

Scrutiny of the cardiac safety of azithromycin intensified in May 2012 with publication of a retrospective observational cohort study evaluating mortality among patients in the Tennessee Medicaid program who received azithromycin, amoxicillin, or no antibiotics. ${ }^{8}$ During the first 5 days of therapy, patients who received azithromycin had an increased risk of cardiovascular death relative to patients who received no antibiotics $(p<0.001)$ and those who received amoxicillin $(p=0.002)$. However, there was no increased risk of cardiovascular death with amoxicillin relative to patients not receiving any antibiotics. The authors estimated that an additional 47 cardiovascular deaths would occur per 1 million courses of azithromycin relative to amoxicillin. $^{8}$

Before clinicians scurry to discontinue azithromycin use for all of their patients, it seems prudent to take a closer look at the Tennessee Medicaid study. ${ }^{8}$ The study design was retrospective and observational, so patients were not randomly assigned to the various groups. Although the patient groups were matched using a propensity score, it is possible that important differences that could affect the study's conclusions still existed between the groups. For example, were the severity of infection and the risk factors for torsades de pointes comparable between the patient groups? The indication for antibiotic use was not recorded for approximately one-third of the patients, so it is not truly known if the groups were similar with regard to the number and types of infections treated. Another concern is that the study results may not be generalizable to other patient populations. The study evaluated persons enrolled in a single state's Medicaid program, but would the results differ in other states or in non-Medicaid patients? Patients had to be regular users of medical care to be included in the cohort. In fact, 4 factors were important determinants of azithromycin use: more than 10 outpatient visits with a noncardiovascular diagnosis in the past year, a prescription for a $ß$-agonist in the past year, an emergency department visit in the past 30 days, or any filled prescription (other than azithromycin) in the past 30 days. Would the study results differ in patients without these important factors? Approximately $75 \%$ of the study cohorts were female, which is a risk factor for torsades de pointes. Would the results have differed if the groups had had an equal distribution of men and women? Lastly, the absolute number of cardiovascular deaths was relatively small. For the 347795 prescriptions of azithromycin over a 15-year period, there were a total of 29 cardiovascular deaths, of which 22 were sudden cardiac deaths. There were 42 cardiovascular deaths (29 sudden cardiac deaths) associated with the 1348672 prescriptions for amoxicillin, and 41 cardiovascular deaths (33 sudden cardiac deaths) among the 1391180 matched controls receiving no antibiotics. ${ }^{8}$ Sudden cardiac deaths were identified using a computerized definition that had an estimated sensitivity of only $75 \%$. Therefore, it is possible that mortality categorized as sudden cardiac death may have had another plausible explanation. Given the inherent weaknesses of retrospective, observational studies and the many unknown factors in this particular study, the excess deaths in the azithromycin group may not have been related to azithromycin use.

In a recent Danish study (published in May 2013), azithromycin was not associated with an increased risk of cardiovascular death when compared with penicillin $\mathrm{V}$, the most commonly used antibiotic in Scandinavia. ${ }^{9}$ In the first 5 days of therapy, there were 17 cardiovascular deaths among the 1102050 episodes of azithromycin use and 146 cardiovascular deaths among the 7364292 episodes of penicillin V use (rate ratio $0.93,95 \%$ confidence interval $[\mathrm{CI}] 0.56-1.55)$. However, this patient population differed from the patients in the study by Ray and others. ${ }^{8}$ Overall, the Danish patients were about a decade younger and had better cardiovascular health than the Tennessee Medicaid patients. ${ }^{10}$

Several large randomized studies have not shown increased mortality among patients receiving azithromycin relative to placebo. ${ }^{11-15}$ In particular, 4 studies evaluated the relative efficacy of azithromycin and placebo in 13500 patients for the secondary prevention of coronary artery disease, based on the hypothesized role of Chlamydophila pneumoniae in atherosclerotic cardiovascular disease. ${ }^{11-14}$ Although these studies did not demonstrate any benefit of azithromycin in preventing secondary coronary events, mortality and cardiovascular death were virtually identical between the azithromycin and placebo groups in these patients with significant cardiac history. In a study evaluating azithromycin for the prevention of acute exacerbations of chronic obstructive pulmonary disease, 1142 patients were randomly assigned to receive azithromycin $250 \mathrm{mg}$ daily or placebo for 1 year. ${ }^{15}$ The rate of death from any cause was 3\% in the azithromycin group and $4 \%$ in the placebo group $(p=0.87)$, and the rate of cardiovascular death was $0.2 \%$ in both groups $(p=1.00)$. However, $6 \%$ of study candidates were excluded at screening because of risk factors for QT prolongation and torsades de pointes, and 10 patients (6 receiving azithromycin, 
4 receiving placebo) were withdrawn from the study 1 month after randomization because their QT interval was greater than $450 \mathrm{~ms}^{16}$

Lastly, macrolides have been shown to significantly decrease mortality in patients with community-acquired pneumonia. Among outpatients and among intubated patients in an intensive care unit, macrolides were associated with significantly lower mortality relative to fluoroquinolones. ${ }^{17,18}$ As is the case for most decisions, clinicians must weigh the benefits of azithromycin against the potential risks. On the basis of all available data, azithromycin remains a viable option for the treatment of community-acquired pneumonia.

\section{References}

1. Samarendra P, Kumari S, Evans SJ, Sacchi TJ, Navarro V. QT prolongation associated with azithromycin/amiodarone combination. Pacing Clin Electrophysiol. 2001;24(10):1572-4.

2. Matsunaga N, Oki Y, Prigollini A. A case of QT-interval prolongation precipitated by azithromycin. NZ Med J. 2003;116(1185):U666.

3. Kim MH, Berkowitz C, Trohman RG. Polymorphic ventricular tachycardia with a normal QT interval following azithromycin. Pacing Clin Electrophysiol. 2005;28(11):1221-2.

4. Huang $\mathrm{BH}, \mathrm{Wu} \mathrm{CH}, \mathrm{Hsia} \mathrm{CP}$, Yin Chen C. Azithromycin-induced torsade de pointes. Pacing Clin Electrophysiol. 2007;30(12):1579-82.

5. Kezerashvili A, Khattak H, Barsky A, Nazari R, Fisher JD. Azithromycin as a cause of QT-interval prolongation and torsade de pointes in the absence of other known precipitating factors. I Interv Card Electrophysiol. 2007; 18(3):243-6.

6. Winton JC, Twilla JD. Sudden cardiac arrest in a patient on chronic methadone after the addition of azithromycin. Am J Med Sci. 2013;345(2):160-2.

7. Poluzzi E, Raschi E, Moretti U, De Ponti F. Drug-induced torsades de pointes: data mining of the public version of the FDA Adverse Event Reporting System (AERS). Pharmacoepidemiol Drug Saf. 2009;18(6):512-8.

8. Ray WA, Murray KT, Hall K, Arbogast PG, Stein CM. Azithromycin and the risk of cardiovascular death. N Engl J Med. 2012;366(20):1881-90.

9. Svanström H, Pasternak B, Hviid A. Use of azithromycin and death from cardiovascular causes. N Engl J Med. 2013;368(18):1704-12.

10. Mosholder AD, Mathew J, Alexander JJ, Smith H, Nambiar S. Cardiovascular risks with azithromycin and other antibacterial drugs. $N$ Engl J Med. 2013;368(18):1665-8.
11. O'Connor CM, Dunne MW, Pfeffer MA, Muhlestein JB, Yao L, Gupta S, et al.; Investigators in the WIZARD Study. Azithromycin for the secondary prevention of coronary heart disease events. The WIZARD study: a randomized controlled trial. JAMA. 2003;290(11):1459-66.

12. Grayston JT, Kronmal RA, Jackson LA, Parisi AF, Muhlestein JB, Cohen JD, et al.; ACES Investigators. Azithromycin for the secondary prevention of coronary events. N Engl J Med. 2005;352(16):1637-45.

13. Muhlestein JB, Anderson JL, Carlquist JF, Salunkhe K, Horne BD, Pearson $\mathrm{RR}$, et al. Randomized secondary prevention trial of azithromycin in patients with coronary artery disease. Primary clinical results of the ACADEMIC study. Circulation. 2000;102(15):1755-60.

14. Cercek B, Shah PK, Noc M, Zahger D, Zehmer U, Matetzky S, et al.; AZACS Investigators. Effect of short-term treatment with azithromycin on recurrent ischaemic events in patients with acute coronary syndrome in the Azithromycin in Acute Coronary Syndrome (AZACS) trial: a randomised controlled trial. Lancet. 2003;361(9360):809-13.

15. Albert RK, Connett J, Bailey WC, Casaburi R, Cooper JAD Jr, Criner GJ, et al.; COPD Clinical Research Network. Azithromycin for prevention of exacerbations of COPD. N Engl J Med. 2011;365(8):689-98.

16. Albert RK, Connett J, Woodruff PG. Azithromycin and the risk of cardiovascular death [letter]. N Engl J Med. 2012;367(8):773-4.

17. Asadi L, Eurich DT, Gamble JM, Minhas-Sandhu JK, Marrie TJ, Majumdar SR. Guideline adherence and macrolides reduced mortality in outpatients with pneumonia. Respir Med. 2012;106(3):451-8.

18. Martin-Loeches I, Lisboa T, Rodriguez A, Putensen C, Annane D, GarnachoMontero J, et al. Combination antibiotic therapy with macrolides improves survival in intubated patients with community-acquired pneumonia. Intensive Care Med. 2010;36(4):612-20.

Michael B Kays, PharmD, FCCP

Associate Professor of Pharmacy Practice

Purdue University College of Pharmacy

Indianapolis, Indiana

Competing interests: Michael Kays has received grant funding from Ortho McNeil for work outside the scope of this article. He serves as an advisory board member and speaker's bureau participant for Forest Labs.

\begin{tabular}{lcc}
\multicolumn{2}{c}{ Advertisers } & \multicolumn{1}{c}{ IndeX } \\
\hline & Ad Page & Prescribing Information \\
\hline Pfizer / Injectables & IFC & - \\
\hline Pfizer / Injectables & 274 & - \\
\hline Pharmaceutical Partners of Canada / Corporate & OBC & - \\
\hline
\end{tabular}

